\title{
BMJ Open Physical-mental multimorbidity in children and youth: a scoping review
}

\author{
Isabella Romano (D) , Claire Buchan, Leonardo Baiocco-Romano, Mark A Ferro
}

To cite: Romano I, Buchan C, Baiocco-Romano L, et al. Physical-mental multimorbidity in children and youth: a scoping review. BMJ Open 2021;11:e043124. doi:10.1136/ bmjopen-2020-043124

- Prepublication history and supplemental material for this paper is available online. To view these files, please visit the journal online (http://dx.doi. org/10.1136/bmjopen-2020043124).

Received 24 July 2020 Accepted 28 April 2021
Check for updates

(C) Author(s) (or their employer(s)) 2021. Re-use permitted under CC BY-NC. No commercial re-use. See rights and permissions. Published by BMJ.

School of Public Health and Health Systems, University of Waterloo, Waterloo, Ontario, Canada

Correspondence to

Dr Mark A Ferro;

mark.ferro@uwaterloo.ca

\begin{abstract}
Objective Efforts to describe the current state of research are needed to advance the field of physical-mental multimorbidity (ie, the co-occurrence of at least one physical illness and at least one mental disorder) among children and youth. Our objective was to systematically explore the breadth of physical-mental multimorbidity research in children and youth and to provide an overview of existing literature topics.

Design Scoping review.

Methods We conducted a systematic search of four key databases: PubMed, EMBASE, PsycINFO and Scopus as well as a thorough scan of relevant grey literature. We included studies of any research design, published in English, referring to physical-mental multimorbidity among children and youth aged $\leq 18$ years. Studies were screened for eligibility and data were extracted, charted and summarised narratively by research focus. Critical appraisal was employed using the modified Quality Index (Ql).
\end{abstract}

Results We included 431 studies and 2 sources of grey literature. Existing research emphasises the co-occurrence of anxiety, mood and attention disorders among children with epilepsy, asthma and allergy. Evidence consists of mostly small, observational studies that use crosssectional data. The average QI score across applicable studies was 9.1 ( $\mathrm{SD}=1.8)$.

Conclusions There is a pressing need for more robust research within the field of child physical-mental multimorbidity.

\section{INTRODUCTION}

While one in four adults will experience mental disorder in their lifetime, approximately one in five individuals will experience mental disorder at some point during childhood or youth. ${ }^{1-3}$ In fact, a majority of cases of adult mental disorder are thought to originate earlier during childhood or adolescence, ${ }^{4-6}$ resulting in substantial burden later in life. ${ }^{7}$ Children and youth are therefore an important population for public health practitioners to consider with respect to preventing mental disorder throughout the life course. ${ }^{8}$ However, the current model of prevention and care may not adequately address children and youth themselves; it is estimated that $75 \%$ of children experiencing symptoms of mental disorder do not receive
Strengths and limitations of this study

This is the first scoping review to map the research in the field of physical-mental multimorbidity (the co-occurrence of at least one physical illness and at least one mental disorder) among children and youth.

- We developed a systematic search strategy for four key electronic databases using Medical Subject Headings, Emtree and Index terms and adapted this strategy for a search of existing grey literature.

- To better describe the existing evidence, we also evaluated the methodological rigour of eligible studies using the modified Quality Index.

- As our systematic search yielded a large number of eligible studies, hand-searching reference lists was not feasible and included studies are not exhaustive; rather, we assess the breadth of existing research.

any specialised services that would be of benefit to them. ${ }^{39}$

Global estimates suggest that approximately one in four children suffer from chronic physical health conditions. ${ }^{10}$ The experience of physical illness can involve considerable challenges imposed by functional limitations as well as effort on behalf of children and their families to overcome these limitations. ${ }^{11}$ Often, experiences of physical illness during childhood span into adulthood ${ }^{12}$ with implications for quality of life throughout the life course. ${ }^{13}$ Moreover, physical illness has been cited as an important, yet overlooked, ${ }^{14}{ }^{15}$ individual-level determinant of mental disorder in children and youth (herein children). ${ }^{11} 16$ In fact, diagnosis of a physical illness may significantly increase the risk of developing a co-occurring mental disorder; approximately $50 \%$ of all children with physical illness will experience poor mental health, ${ }^{17}$ where as many as $20 \%-30 \%$ may meet criteria for a clinical diagnosis. ${ }^{101518}$

An estimated $15 \%$ of all children will experience the co-occurrence of at least one physical illness and at least one mental disorder-defined as physical-mental multimorbidity. ${ }^{19}$ Physical-mental multimorbidity is often chronic and has been shown to be 
associated with significant negative impacts on daily functioning, ${ }^{20} 21$ increases in mental health service use ${ }^{22}$ and greater likelihood of suicidality ${ }^{23}$ and risky substance use $^{2425}$ among youth. However, despite increased interest in child mental health in recent decades as well as modern advancements in healthcare for children with physical illnesses, research on child physical-mental multimorbidity remains limited. Present literature widely emphasises adult and geriatric populations and, moreover, much of the existing literature addressing the co-occurrence of physical illness and mental disorder among children has likely not yet been classified as research on 'multimorbidity' per se, but rather 'comorbidity'. Comorbidity refers to the effects of additional conditions on a single index condition, ${ }^{26}{ }^{27}$ in contrast, multimorbidity refers to two or more co-occurring conditions but without necessary reference to an index condition. ${ }^{26}$ Physical-mental multimorbidity therefore indicates that no single physical illness or mental disorder holds priority with respect to an individual's healthcare and well-being. ${ }^{26}$

Efforts to describe the current state of research are needed to advance the field of physical-mental multimorbidity among children. As such, this scoping review aimed to provide an overview of existing literature with the following objectives to: (1) conduct a systematic search of peer-reviewed and grey literature on child physical-mental multimorbidity; (2) map study characteristics across existing literature; (3) summarise research topics addressed to date; (4) critically appraise existing research, where applicable and (5) identify limitations and gaps noted within current evidence. Results gathered and recommendations made from this scoping review are intended to guide future research in child physicalmental multimorbidity.

\section{METHODS}

This scoping review follows the six-stage framework proposed by Arksey and $\mathrm{O}^{\prime}$ Malley ${ }^{28}$ and enhanced by Levac and colleagues. ${ }^{29}$ We discuss each stage briefly below, whereas a detailed peer-reviewed protocol is available in print. ${ }^{30}$ Reporting follows the PRISMA Extension for Scoping Reviews checklist. ${ }^{31}$

\section{Identifying the research questions}

We used the following research question to guide this scoping review: 'What is known about child physicalmental multimorbidity?'. We identified secondary research questions to further deepen the understanding of existing literature and direct the reporting of results. ${ }^{30}$ These included: 'What types of research on child and youth physical-mental multimorbidity have been conducted?', 'What physical illnesses and mental disorders have been investigated?' and 'What identifiable questions have yet to be addressed within the literature?' To answer these research questions, we also relied on the following definitions: (1) physical illness referred to biologically based chronic conditions, characterised by functional limitations ${ }^{11}$ and (2) mental disorder was defined according to the Diagnostic and Statistical Manual of Mental Disorders (DSM) classification of conditions reflecting dysfunction in the "psychological, biological, or developmental processes underlying mental functioning, ${ }^{32}$

\section{Identifying relevant studies}

We developed and implemented a systematic search to identify relevant studies across four electronic databases: PubMed, EMBASE, PsycINFO and Scopus. The following search was initially developed for PubMed using Medical Subject Heading (MeSH) terms:

("chronic disease"[MeSH Terms] OR comorbidity[MeSH Terms]) AND ("anxiety disorders"[MeSH Terms] OR "mood disorders"[MeSH Terms] OR "disruptive, impulse control, and conduct disorders"[MeSH Terms] OR "attention deficit and disruptive behavior disorders" [MeSH Terms]) AND (English[lang] AND (("infant"[MeSH Terms] OR "child"[MeSH Terms] OR "adolescent"[MeSH Terms]) OR "child"[MeSH Terms] OR "adolescent"[MeSH Terms] OR "young adult"[MeSH Terms])) NOT (adult[MESH:noexp] OR aged[mesh:noexp])

Notably, we relied on the MeSH term 'comorbidity' rather than 'multimorbidity' for two reasons: (1) in recognition that much of the existing research was likely conducted within the context of an index condition and (2) at the time of this study, 'multimorbidity' was not a MeSH term. The search was adapted using Emtree terms in EMBASE and Index terms in PsycINFO; Scopus does not have a controlled vocabulary of its own but includes subject headings from other databases. Our systematic search strategy was developed with consultation from a subject-specialty liaison librarian at our institution.

We conducted the initial search on 30 April 2018 with an update on 19 March 2020. Citations were exported into Mendeley reference management software. Duplicates were automatically deleted by the software prior to screening.

As described in our protocol, ${ }^{30}$ we also conducted a search for relevant grey literature to ensure that our review captured all potentially relevant material on child physical-mental multimorbidity. Grey literature is differentiated as being published outside of traditional academic routes and is usually produced by organisations or governments. ${ }^{33}$ These differed from other sources retrieved through our systematic search, regardless of peer-review status (eg, commentaries, dissertations). We relied on two existing custom Google search engines for grey literature: one for Canadian public health information $^{34}$ and another for Canadian and international government publications. ${ }^{35}$

\section{Selecting studies}

Study selection was conducted in two sequential steps: (1) by title and abstract and (2) by full-text review, each by 
two separate reviewers. All studies referring to physicalmental multimorbidity as defined here (ie, co-occurrence of at least one physical illness and at least one mental disorder) among children or youth (ie, $\leq 18$ years of age) were included. Additional inclusion criteria were publication in English and evidence of peer-review (with exceptions to grey literature, commentaries and dissertations). Studies of any design were to be included. Discrepancies between reviewers were resolved through third-party consensus when required.

\section{Charting the data}

Reviewers were each assigned studies selected for final inclusion. Key study characteristics were collected using a data extraction tool previously described in the protocol. ${ }^{30}$ Data were entered and stored in a custom-built Microsoft Excel database linked to the data extraction tool. Extracted information included bibliometric (eg, year of publication, country, publication type), epidemiological (eg, study design, time to follow-up) and population (eg, age, sex) characteristics pertaining to the included studies, in addition to the relevant research foci (ie, physical illness(es), mental disorder(s)) across studies.

Independent reviewers were assigned studies for critical appraisal. We used the modified version of the Quality Index (QI), ${ }^{36}$ developed by Ferro and Speechley, ${ }^{37}$ to assign a methodological quality score to all relevant original research studies. This exercise was intended to strengthen our examination of the scope of current evidence by allowing us to further describe studies according to the methodological details they reported. Each study was assigned a numeric score ranging from 0 to 15 , calculated where 'yes' $=1$ and 'no' $=0$ across the following domains: (1) reporting (items 1-7); (2) external validity (items $8-10$ ); (3) internal validity (items 11-14) and (4) study power (item 15). ${ }^{37}$ Higher scores indicated higher quality.

\section{Collating, summarising and reporting the results}

Data collected through charting were collated, summarised and reported herein to map the breadth of research on child physical-mental multimorbidity. Consistent with Arksey and O'Malley's scoping review framework, ${ }^{28}$ results were presented narratively and in two ways. First is by describing the characteristics of charted literature. Second is by organising the literature thematically according to (a) illness focus and (b) cited recommendations and limitations within the context of existing physical-mental multimorbidity research. The aim of this stage in the scoping review process was to provide an overview of existing literature in an attempt to identify gaps in knowledge and inform future research.

\section{RESULTS}

\section{Study selection}

The systematic database searches yielded a total of 10561 non-duplicate studies. At the first screening step involving titles and abstracts, 9772 were excluded for failing to meet inclusion criteria. Seven-hundred and eighty-nine articles were retrieved for full-text review, after which 358 studies were eliminated. A total of 431 articles were selected for data extraction and charting. Figure 1 summarises the review process using a CONSORT flow diagram. The grey literature search yielded two sources. Refer to online supplemental file A for a reference list of all included studies and grey literature.

\section{Description of child and youth physical-mental multimorbidity studies}

Extracted study characteristics are summarised in table 1 and described in more detail below. The full scoping review dataset is available to readers on request to the authors or through accessing the Dryad data repository. ${ }^{38}$

\section{Bibliometric characteristics}

Publication year ranged from 1991 to 2020. More than half $(n=281,65 \%)$ of studies were published within the past decade. Studies from across six continents (ie, Africa, Asia, Australia, Europe, North America and South America) were included; nearly half $(n=205,48 \%)$ were from North America, with the majority having been conducted in the USA. The majority of publications represented original research $(\mathrm{n}=268)$; an additional 75 were synthesised review articles, 38 were reports (19 case studies, 13 commentaries) and 51 were peer-reviewed abstracts and conference proceedings.

\section{Epidemiological characteristics}

Among the included studies, 250 (58\%) were crosssectional in design while $72(17 \%)$ were longitudinal (55 prospective cohort; 17 retrospective cohort). Interval to follow-up among longitudinal studies ranged from 5 days to 17 years, but the majority of studies involving $>1$ timepoint had an average interval of less than 1 year between baseline and follow-up. Of the 75 synthesised review articles, 51 were narrative reviews, 16 were systematic reviews and 10 were meta-analyses; the majority of syntheses were centred around one index condition.

\section{Population characteristics}

As per the objective of this scoping review, all participants from the included studies were children and/or youth aged 18 years or younger. In 65 studies, participants were aged less than 12 years and 43 studies included adolescents only (ages 13-18 years). The majority of studies $(n=298,69 \%)$ included multiple child age groups. Studies predominately included both male and female $(\mathrm{n}=354,82 \%)$ children and youth, but 49 did not specify a study population sex. Some studies reported findings from only male $(n=19)$ or female $(n=9)$ participants. Sample sizes within original research studies varied greatly across study populations-between 10 and 1125 161 participants-although the median sample size was 177.5 . 


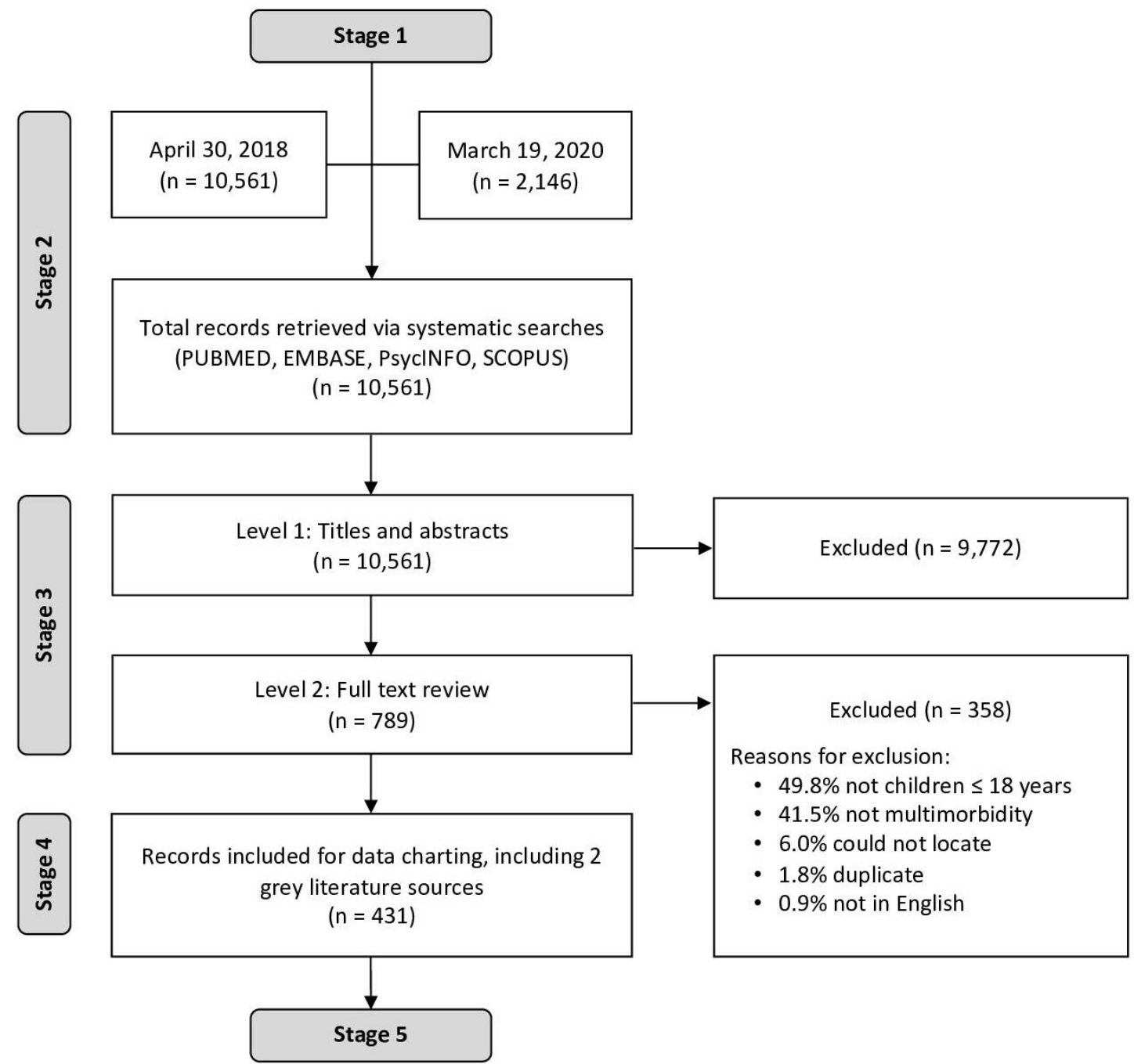

Figure 1 CONSORT flow diagram depicting the record selection process through different stages of the scoping review.

\section{Quality Index scoring}

Among studies eligible for quality indexing ( $\mathrm{n}=270$; that is, original quantitative studies), the overall average QI score was $9.1(\mathrm{SD}=1.8)$; scores ranged from 3 to 13 . Mean scores across specific subsections were $4.9(\mathrm{SD}=0.5$; range: $0-7)$ for reporting, $1.1(\mathrm{SD}=0.5$; range: $0-3)$ for external validity and 3.1 ( $\mathrm{SD}=0.4$; range: $0-4)$ for internal validity. Sixteen studies provided sample size or power calculations to detect important effects.

\section{Research foci of child and youth physical-mental multimorbidity literature}

We selected literature examining physical-mental multimorbidity across a wide variety of physical illnesses and mental disorders among children and youth. These studies largely focused on one physical illness at a time and its co-occurrence with at least one mental disorder. As such, primary research foci were grouped into themes accordingly: first, by physical illness and then by mental disorder; these are presented in the balloon plot shown in figure 2, where larger bubbles indicate greater representation within the literature. Note that because physical illness categories were not mutually exclusive across studies, totals shown do not sum to 431; this is also true for mental disorder categories.

While a range of physical illnesses were cited, there appeared to be extensive overlap in the co-occurring mental disorders of focus. The most common physical illnesses included in the literature were epilepsy $(n=136)$, asthma $(n=75)$, chronic pain (including headache and migraine, $n=69)$, allergic disorders $(n=52)$ and diabetes mellitus $(n=46)$. Among mental disorders, these predominately included anxiety disorders $(\mathrm{n}=270)$, attention deficit hyperactivity disorder (ADHD) $(\mathrm{n}=260)$ and mood disorders $(n=245)$. Behavioural disorders such as conduct disorder $(n=80)$ and other disorders (eg, schizoaffective disorders, psychosis, eating disorders; $n=42$ ) accounted for a smaller proportion of cited mental disorders. A total of 63 studies $(14.6 \%)$ referred to multiple physical illnesses and 243 studies (56.4\%) referred to multiple mental disorders. A full narrative description across foci is available in online supplemental file B; across all studies, findings generally indicated high rates of multimorbidity within the context of mental disorders among children with physical illness. Moreover, studies commonly noted 
Table 1 Bibliometric, epidemiological and population characteristics among child physical-mental multimorbidity studies included in the present scoping review $(\mathrm{N}=431)$

\begin{tabular}{cll}
\hline & N & $\%$ \\
\hline $\begin{array}{l}\text { Publication year } \\
1991-1995\end{array}$ & 7 & \\
$1996-2000$ & 7 & 1.6 \\
$2001-2005$ & 38 & 1.6 \\
$2006-2010$ & 98 & 8.8 \\
$2011-2015$ & 140 & 32.7 \\
$2016-2020$ & 141 & 32.7
\end{tabular}

Continent

\begin{tabular}{|lll|}
\hline Africa & 9 & 2.1 \\
\hline Asia & 67 & 15.5 \\
\hline Australia & 12 & 2.8 \\
\hline Europe (including UK) & 125 & 29.0 \\
\hline North America & 205 & 47.6 \\
\hline South America & 13 & 3.0 \\
\hline Publication type & & \\
\hline Abstract & 51 & 11.8 \\
\hline Dissertation & 1 & 0.2 \\
\hline Original research & 268 & 62.2 \\
\hline Protocol & 1 & 0.2 \\
\hline Report & 35 & 8.1 \\
\hline Review & 75 & 17.4 \\
\hline
\end{tabular}

Study design

\begin{tabular}{|lll|}
\hline Case report/series & 19 & 4.4 \\
\hline Commentary & 13 & 3.0 \\
\hline Cross-sectional & 250 & 58.0 \\
\hline Meta-analysis & 10 & 2.3 \\
\hline Narrative review & 51 & 11.8 \\
\hline Prospective & 55 & 12.8 \\
\hline Retrospective & 17 & 3.9 \\
\hline Systematic review & 16 & 3.7 \\
\hline Time to follow-up* & & \\
\hline Range & 5 days to 17 & - \\
\hline Mean (months, SD) & years & \\
\hline$\leq 1$ year & 47.2 & 54.0 \\
\hline $1-5$ years & 26 & 43.3 \\
\hline $5-10$ years & 17 & 28.3 \\
\hline$>10$ years & 11 & 18.3 \\
\hline Sample size $\dagger$ & 6 & 10.0 \\
\hline Range $(n)$ & & \\
\hline Median $(n)$ & $10-1125161$ & - \\
\hline Mean $(n, S D)$ & 177.5 & - \\
\hline$\leq 50$ & 22543.7 & 107482.2 \\
\hline $50-100$ & 35 & 13.1 \\
\hline & 57 & 21.3 \\
\hline
\end{tabular}

Continued
Table 1 Continued

\begin{tabular}{|c|c|c|}
\hline & $\mathbf{N}$ & $\%$ \\
\hline $100-250$ & 60 & 22.4 \\
\hline $250-1000$ & 39 & 14.6 \\
\hline $1000-10000$ & 37 & 13.8 \\
\hline$>2000$ & 40 & 14.9 \\
\hline \multicolumn{3}{|l|}{ Age group } \\
\hline Children only ( $\leq 12$ years) & 65 & 15.1 \\
\hline Adolescents only (>13 years) & 43 & 10.0 \\
\hline Both ( $\leq 18$ years) & 298 & 69.1 \\
\hline Not reported & 25 & 5.8 \\
\hline \multicolumn{3}{|l|}{ Sex/gender } \\
\hline Females only & 9 & 2.1 \\
\hline Males only & 19 & 4.4 \\
\hline Both & 354 & 82.1 \\
\hline Not specified & 49 & 1.4 \\
\hline
\end{tabular}

*Among original research studies of prospective or retrospective design (abstracts and case reports/series excluded) in which time to follow-up was reported $(n=60)$.

†Original research only (abstracts and case reports/series excluded; $n=268$ ).

significant effects on health-related quality of life for patients, highlighting the implications of physical-mental multimorbidity on patients' well-being.

\section{Grey literature}

The grey literature search retrieved a total of 34 sources (duplicates deleted), two of which were relevant to child physical-mental multimorbidity upon full text review. Sources were deleted if they were not reflective of physicalmental multimorbidity $(\mathrm{n}=25)$ or not specific to children $(\mathrm{n}=7)$. One public health report addressed the potentially reciprocal and bidirectional relationship between obesity and mental health problems including anxiety and depression. ${ }^{39}$ A school-based guide to promoting student mental health included information for educators that anxiety in children may manifest as physical pain and headache. ${ }^{40}$

\section{Patient and public involvement}

Patients or the public were not involved in the design, or conduct, or reporting, or dissemination plans of our research.

\section{DISCUSSION}

This scoping review included 431 sources of evidence on physical-mental multimorbidity among children and youth as a result of a systematic search of the peer-reviewed and grey literature. Our findings demonstrate the extant breadth of physical-mental multimorbidity research characterised by a rapid increase in studies published over the past decade. However, it is clear through our review that 


\begin{tabular}{|c|c|c|c|c|c|c|c|c|c|c|c|}
\hline \multirow[b]{2}{*}{ Physical illness } & \multicolumn{10}{|c|}{ Mental disorder } & \multirow{3}{*}{$\begin{array}{c}\text { Total: } \\
52\end{array}$} \\
\hline & Anxiety & & Mood & & ADHD & & Behavio & & Other & & \\
\hline Allergic disorders & & 26 & & 23 & & 38 & O & 12 & - & 7 & \\
\hline Asthma & & 54 & & 42 & & 35 & O & 16 & - & 3 & 75 \\
\hline Cardiac disorders & & 12 & - & 9 & & 13 & - & 7 & - & 1 & 20 \\
\hline Cerebral palsy & 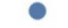 & 9 & 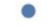 & 6 & 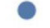 & 11 & - & 4 & - & 1 & 12 \\
\hline Chronic pain, headache, migraine & & 61 & & 53 & & 33 & 0 & 20 & - & 4 & 69 \\
\hline Cystic fibrosis & 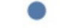 & 10 & 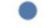 & 11 & $\bullet$ & 2 & - & 3 & - & 1 & 13 \\
\hline Diabetes mellitus & & 34 & & 33 & & 20 & - & 10 & - & 7 & 46 \\
\hline Epilepsy & & 76 & & 72 & & 102 & 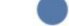 & 28 & - & 11 & 136 \\
\hline Gastrointestinal disorders & & 27 & & 27 & & 17 & - & 9 & - & 6 & 38 \\
\hline Hematologic disorders & & 13 & 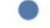 & 12 & - & 6 & - & 6 & - & 1 & 17 \\
\hline Musculoskeletal disorders & & 21 & & 19 & & 18 & 0 & 17 & - & 3 & 28 \\
\hline Obesity & & 19 & & 19 & & 31 & - & 5 & - & 5 & 41 \\
\hline Pediatric cancer & 0 & 12 & 0 & 13 & $\bullet$ & 3 & - & 3 & - & 2 & 19 \\
\hline Other & & 28 & & 24 & & 34 & - & 10 & - & 10 & 51 \\
\hline Total: & & & 45 & & 260 & & 80 & & 42 & & \\
\hline
\end{tabular}

Figure 2 Research foci across child physical-mental multimorbidity studies included in the present scoping review. ADHD, attention deficit hyperactivity disorder.

most of the existing research is likely classified as comorbidity within the literature, usually, according to a single physical illness index condition in relation to multiple mental disorders. This highlights a need for broader research on the impact of physical-mental multimorbidity on children and youth.

In order to provide an overview of existing literature on physical-mental multimorbidity in children and youth, we primarily sought to examine the extent, range and nature of research activity within this field. ${ }^{28}$ As far as we are aware, this scoping review represents the first published synthesis of research in this field. Given the complexity introduced by lack of consistent terminology, mapping the state of existing research was an important first step to advancing the field and directing future research. The resulting product includes a compendium of published topics within physical-mental multimorbidity research among children (figure 2; online supplemental file B).

Findings from this scoping review suggest that much of the focus within the existing physical-mental multimorbidity literature includes ADHD, anxiety and mood disorders among children with epilepsy, asthma and allergy. We anticipated this finding given that the distribution of physical illness categories appeared to be generally proportionate to observed population prevalence rates and the associated burden to child health. However, it was perhaps a surprise that the systematic search strategy also yielded a considerable number of studies related to mental disorder among children with obesity and with chronic non-cancer pain (including headache and migraine). Future research should consider these conditions as within the scope of physical-mental multimorbidity. To a lesser extent, some research had also been conducted among child populations with relatively rarer physical illnesses including inflammatory bowel diseases, sickle cell disease, cerebral palsy, cardiac disorders, childhood cancer and cystic fibrosis, largely also in relation to ADHD, anxiety and mood disorders. Given the lack of prior syntheses, the extent to which existing research has addressed physical-mental multimorbidity within child populations was previously unclear. Our scoping review was intended to map this field of research, as it was previously difficult to describe to describe the range of material that had been published.

Bibliometric, population characteristic and epidemiological data were extracted from included studies for the purpose of describing the main sources and types of evidence that exist. Findings indicated that the existing evidence comes largely from observational studies using cross-sectional data collected among children and youth from North America and Europe. Sample sizes among studies varied greatly, but few studies provided sample size or power calculations. Predominate conclusions made across the literature included generally high prevalence of co-occurring mental disorders among children with physical illness, and the effects of physical-mental multimorbidity on health-related quality of life and wellbeing among children and youth.

We incorporated the modified $\mathrm{QI}^{3637}$ as a novel methodological component-strengthening the rigour of our scoping review. We made critical appraisal an objective for the purpose of further describing the existing research available on child physical-mental multimorbidity; we sought to gain deeper insight into the methodological quality of existing evidence. Findings indicated a midrange quality score across all studies, suggesting a need for evidence from studies with more robust methods. Evaluation of external validity indicated that it may be difficult to generalise findings from much of the current child physical-mental multimorbidity literature. On the 
other hand, the existing evidence may generally represent adequate internal validity. Future research should build on these gaps in methodological quality to produce more robust evidence that is applicable to broader and more diverse subpopulations of children with physical illness. Unlike systematic reviews, critical appraisal is not a typical component of scoping reviews and, to our knowledge, this is the first scoping review to adopt the modified QI as outlined by Ferro and Speechley. ${ }^{37}$ Future studies may seek to delve deeper into the empirical evidence of child physical-mental multimorbidity through systematic reviews and meta-analyses where applicable, and where their research questions are considerably more focused than that of a scoping review. ${ }^{41}$ For example, we recommend that subsequent evidence syntheses aim to systematically estimate and compare the prevalence of physical-mental multimorbidity among population subgroups of children; assessing and pooling the observations made within individual studies should be made a priority for future research, as doing so was beyond the scope of our current review.

We also sought to incorporate a scan of potential grey literature in our scoping review, as grey literature has been recognised as an important source of information for review syntheses. ${ }^{42}$ Our search results yielded only two sources, possibly indicative of limited knowledge translation efforts within an already limited body of research on child physical-mental multimorbidity. In this regard, searching the grey literature did not contribute substantially to our current scoping review findings. However, we encourage other researchers to consider including grey literature in their scoping reviews; these sources can present important information that has already been disseminated to patients and knowledge users by organisations and government agencies. Researchers may wish to expand their search to other databases and search engines.

\section{Gaps in research on child physical-mental multimorbidity}

We identified gaps in the existing literature in an effort to guide future research in child physical-mental multimorbidity. First, it remains clear that additional research is needed among foci related to rarer conditions, specifically where fewer studies were yielded by our systematic search. While under-representation of some physical illnesses and mental disorders in the literature were likely reflective of relatively lower population prevalence, additional research is warranted. Second, previous work suggests that regardless of specific diagnoses, the risk of mental disorder for children with physical illnesses may be relatively consistent ${ }^{17}{ }^{43}$ and indicative of common underlying risk factors. As such, we identify a need for studies that examine multiple illnesses to allow for crossillness comparisons. A broader focus on physical-mental multimorbidity is required, whereas much of the existing research is siloed into populations of children and adolescents with one physical illness. Third, there remain a paucity of studies addressing the underlying causal mechanisms of physical-mental multimorbidity in children, and a lack of evidence regarding predictors. Future research should seek to explore potential causal relationships between physical illness and mental disorder among children, including physiological and psychosocial mechanisms of onset. Continued research on possible correlates, predictors and sequelae among children with physical-mental multimorbidity is also required. Fourth, inconsistent data sources and collection methods were observed and noted by our study team. Inquiry into the impact of assessment variation is needed, as assessment type (eg, standardised interviews, problem checklists) may influence prevalence estimates in children. ${ }^{44}$ Furthermore, inclusion of multidimensional methods-for example, by engaging both children and their parents-may improve the quality of evidence; previous work has shown discrepancies in child and parental reports. ${ }^{45}{ }^{46}$ Fifth, there was a clear gap in the literature regarding findings from qualitative studies. Future research should aim to study child physical-mental multimorbidity through a qualitative lens to better understand the experiences of children and their parents. Last, more detailed epidemiological research surrounding the impact of child physical-mental multimorbidity on the health system is needed. Much of the existing literature relates to prevalence of mental disorder among children with some physical illness, while there appeared to be less evidence relating to health service utilisation. In light of these gaps, additional research is needed; the Multimorbidity in Children and Youth Across the Life-course ${ }^{19}$ prospective study, for example, will address several of the recommendations and limitations cited among studies included in our present scoping review.

\section{Strengths and limitations}

The purpose of adopting a scoping review methodology is generally to examine breadth rather than depth of a given research topic. ${ }^{28}$ While this limitation is balanced by our inclusion of critical appraisal, the level of detail we were able to incorporate into the synthesis of results was limited. Most notably, it was not within the scope of this study to evaluate the findings of included literature, or to incorporate statistical analyses; future research syntheses (eg, systematic reviews, meta-analyses) should do this by addressing more specific research questions. Whereas systematic reviews are also meant to provide complete and exhaustive summaries of existing evidence, we were limited to providing a broader perspective on the field of child physical-mental multimorbidity through a high-level narrative description of available research. It is also possible that the list of studies we retrieved do not exhaust the existing literature. Our search yielded a large number of studies, and hand-searching reference lists was not feasible. In addition, while findings are up to date as of 19 March 2020, they only reflect publications that have been indexed and assigned MeSH, Emtree or Index terms. Although we used multiple databases to increase the robustness of our systematic search, we cannot assume that database indexing procedures are 
completely reliable. This limitation may pose implications for the sensitivity of our systematic search and therefore also the validity of our results. Last, we acknowledge potential inefficiencies in our systematic search strategy. We purposefully used a broad search strategy and relatively loose screening criteria; as a result, more than $90 \%$ of studies did not pass the first stage of abstract and title review. We recommend future syntheses benefit from more targeted searches.

\section{Protocol deviations}

Last, we wish to briefly outline any instances where the current study deviated from the original scoping review protocol. ${ }^{30}$ First, our data extraction tool was slightly revised. We omitted extracting 'Study Type' from each source of evidence as it became redundant on review. Instead, the revised extraction tool included specific categories for study designs (consisting of case report, case series, commentary, cross-sectional, meta-analysis, narrative review, prospective, retrospective and systematic review). Moreover, as they were difficult to decipher and accurately label during data extraction, we did not record any International Classificaton of Diseases (ICD) or DSM codes for physical illnesses or mental disorders. Instead, conditions were extracted using their original wording, and the research team used ICD and DSM categories as guides for grouping studies by physical illness and mental disorder type. Second, as previously noted, we did not hand-search reference lists. Although we initially intended to do so, this became impractical given the size of our scoping review; however, we remain confident that the included literature (although not exhaustive) adequately represents the existing body of child physical-mental multimorbidity evidence. Third, our protocol specified a stringent study timeline which was not feasible. We caution other researchers from underestimating the amount of time and resources needed to conduct a robust scoping review.

\section{CONCLUSION}

This scoping review sought to map the current literature on physical-mental multimorbidity among children and youth through a systematic search of peer-reviewed and grey literature. Results indicate that the past decade has seen a rapid increase in the number of physicalmental multimorbidity research articles published, with emphasis placed on comorbid attention deficit hyperactivity disorder, anxiety and depression in children with epilepsy, asthma and allergy. Existing evidence mostly includes small, observational studies that use crosssectional data collected from North American and European children. Studies yielded through our search were unlikely to be classified as multimorbidity research and were of moderate methodological quality. This is the first scoping review conducted on child physical-mental multimorbidity. Our findings suggest a need for more robust research within the field.
Acknowledgements The authors would like to thank and acknowledge the following individuals for their contributions to this study: (1) Mss Irina I Oltean, Madeline Reed and Sydney Whitney for collecting data and proofreading the manuscript prior to submission; (2) Ms Amanda Doggett for providing data visualisation consultation and (3) Ms Jackie Stapleton who provided valuable guidance and helpful insight throughout the planning and execution of this study. Dr Ferro holds the Canada Research Chair in Youth Mental Health and Early Researcher Award from the Ministry of Research, Innovation and Science.

Contributors All authors contributed meaningfully to the preparation, drafting and editing of this study. IR designed and led the study, collected data, performed the synthesis and analysis and drafted the full manuscript. CB aided in developing the systematic database search framework, collected data, drafted the methods section and critically reviewed the manuscript. LB-R collected data, drafted the introduction section and critically reviewed the manuscript. MAF (guarantor, corresponding author) conceived the manuscript idea, supervised the research and revised the manuscript for critical content. All authors approved the final submitted manuscript and agree to be accountable for all aspects of this study.

Funding The authors have not declared a specific grant for this research from any funding agency in the public, commercial or not-for-profit sectors.

Competing interests None declared.

Patient consent for publication Not required.

Provenance and peer review Not commissioned; externally peer reviewed.

Data availability statement Data are available in a public, open access repository and can be accessed via the Dryad data repository at http://datadryad.org/ with the doi:10.5061/dryad.12jm63xw0.

Supplemental material This content has been supplied by the author(s). It has not been vetted by BMJ Publishing Group Limited (BMJ) and may not have been peer-reviewed. Any opinions or recommendations discussed are solely those of the author(s) and are not endorsed by BMJ. BMJ disclaims all liability and responsibility arising from any reliance placed on the content. Where the content includes any translated material, BMJ does not warrant the accuracy and reliability of the translations (including but not limited to local regulations, clinical guidelines, terminology, drug names and drug dosages), and is not responsible for any error and/or omissions arising from translation and adaptation or otherwise.

Open access This is an open access article distributed in accordance with the Creative Commons Attribution Non Commercial (CC BY-NC 4.0) license, which permits others to distribute, remix, adapt, build upon this work non-commercially, and license their derivative works on different terms, provided the original work is properly cited, appropriate credit is given, any changes made indicated, and the use is non-commercial. See: http://creativecommons.org/licenses/by-nc/4.0/.

\section{ORCID iD}

Isabella Romano http://orcid.org/0000-0002-9322-4331

\section{REFERENCES}

1 Perou R, Bitsko RH, Blumberg SJ, et al. Mental health surveillance among children--United States, 2005-2011. MMWR Supp/ 2013:62:1-35.

2 McMartin SE, Kingsbury M, Dykxhoorn J, et al. Time trends in symptoms of mental illness in children and adolescents in Canada. CMAJ 2014;186:E672-8.

3 Georgiades K, Duncan L, Wang L, et al. Six-Month prevalence of mental disorders and service contacts among children and youth in Ontario: evidence from the 2014 Ontario child health study. Can J Psychiatry 2019;64:246-55.

4 Kessler RC, Berglund P, Demler O, et al. Lifetime prevalence and age-of-onset distributions of DSM-IV disorders in the National comorbidity survey replication. Arch Gen Psychiatry 2005;62:593-602.

5 Westerhof GJ, Keyes CLM. Mental illness and mental health: the two continua model across the lifespan. J Adult Dev 2010;17:110-9.

6 Woodward LJ, Fergusson DM. Life course outcomes of young people with anxiety disorders in adolescence. J Am Acad Child Adolesc Psychiatry 2001;40:1086-93.

7 Waddell C, Schwartz C, Andres C. Making children's mental health a public policy priority: For the one and the many. Public Health Ethics 2018;11:191-200.

8 Waddell C, Georgiades K, Duncan L, et al. 2014 Ontario child health study findings: policy implications for Canada. Can J Psychiatry 2019;64:227-31. 
9 Waddell C, McEwan K, Shepherd CA, et al. A public health strategy to improve the mental health of Canadian children. Can J Psychiatry 2005;50:226-33.

10 Merikangas KR, Calkins ME, Burstein M, et al. Comorbidity of physical and mental disorders in the neurodevelopmental genomics cohort study. Pediatrics 2015;135:e927-38.

11 van der Lee JH, Mokkink LB, Grootenhuis MA, et al. Definitions and measurement of chronic health conditions in childhood: a systematic review. JAMA 2007;297:2741-51.

12 Kuh D, Shlomo YB, eds. A life course approach to chronic disease epidemiology. Oxford University Press, 2004.

13 Moreira H, Carona C, Silva N, et al. Psychological and quality of life outcomes in pediatric populations: a parent-child perspective. $J$ Pediatr 2013;163:1471-8.

14 Bai G, Herten MH-van, Landgraf JM, et al. Childhood chronic conditions and health-related quality of life: findings from a large population-based study. PLoS One 2017;12:e0178539.

15 Ferro MA. Major depressive disorder, suicidal behaviour, bipolar disorder, and generalised anxiety disorder among emerging adults with and without chronic health conditions. Epidemiol Psychiatr Sci 2016;25:462-74.

16 Merikangas KR, Nakamura EF, Kessler RC. Epidemiology of mental disorders in children and adolescents. Dialogues Clin Neurosci 2009;11:7.

17 Butler A, Van Lieshout RJ, Lipman EL, et al. Mental disorder in children with physical conditions: a pilot study. BMJ Open 2018;8:e019011.

18 Tegethoff M, Belardi A, Stalujanis E, et al. Association between mental disorders and physical diseases in adolescents from a nationally representative cohort. Psychosom Med 2015;77:319-32.

19 Ferro MA, Lipman EL, Van Lieshout RJ, et al. Multimorbidity in children and youth across the life-course (my life): protocol of a Canadian prospective study. BMJ Open 2019;9:e034544.

20 Reaume SV, Ferro MA. Chronicity of mental comorbidity in children with new-onset physical illness. Child Care Health Dev 2019;45:559-67.

21 Qadeer RA, Shanahan L, Ferro MA. Chronic disruptive pain in emerging adults with and without chronic health conditions and the Moderating role of psychiatric disorders: evidence from a populationbased cross-sectional survey in Canada. Scand J Pain 2017;17:30-6.

22 Reaume SV, Luther AWM, Ferro MA. Physical morbidity and mental health care among young people. J Adolesc Health 2021;68:540-7.

23 Ferro MA, Rhodes A, Kimber M. Suicidal behaviour among young people with self-reported chronic illness. Can J Psychiatry 2017;62:845-53.

24 Luther AWM, Reaume SV, Qadeer RA, et al. Substance use disorders among youth with chronic physical illness. Addict Behav 2020;110:106517.

25 Qadeer RA, Wong FC, MacKillop J, et al. A comparative study of substance use in young adolescents with and without chronic health conditions. J Child Adolesc Subst Abuse 2018;27:297-304.

26 Nicholson K, Makovski TT, Griffith LE, et al. Multimorbidity and comorbidity revisited: Refining the concepts for international health research. J Clin Epidemiol 2019;105:142.

27 van den Akker M, Buntinx F, Knottnerus JA. Comorbidity or multimorbidity: what's in a name? A review of literature. Eur $J$ Gen Pract 1996;2:65-70. doi:10.3109/13814789609162146
28 Arksey H, O'Malley L. Scoping studies: towards a methodological framework. Int J Soc Res Methodol 2005;8:19-32.

29 Levac D, Colquhoun H, O'Brien KK. Scoping studies: advancing the methodology. Implement Sci 2010;5:69.

30 Romano I, Buchan MC, Ferro MA. Multimorbidity in children and youth: a scoping review protocol. BMJ Open 2018;8:e022413.

31 Tricco AC, Lillie E, Zarin W, et al. PRISMA extension for scoping reviews (PRISMA-ScR): checklist and explanation. Ann Intern Med 2018;169:467-73.

32 American Psychiatric Association (APA). Diagnostic and statistical manual of mental disorders. 5th ed. Washington, DC: APA, 2013.

33 Paez A. Gray literature: an important resource in systematic reviews. J Evid Based Med 2017;10:233-40.

34 Ontario Public Health Libraries Association. Custom search engine for Canadian public health information, 2015. Available: http://www. ophla.ca/customsearchcanada.htm [Accessed 19 Mar 2020].

35 Maps, Data, and Government Information Centre. Search for government publications in primo and Google custom search, 2015. Available: http://subjectguides.uwaterloo.ca/content.php?pid= 408339 [Accessed 19 Mar 2020].

36 Downs $\mathrm{SH}$, Black $\mathrm{N}$. The feasibility of creating a checklist for the assessment of the methodological quality both of randomised and non-randomised studies of health care interventions. J Epidemiol Community Health 1998;52:377-84.

37 Ferro MA, Speechley KN. Depressive symptoms among mothers of children with epilepsy: a review of prevalence, associated factors, and impact on children. Epilepsia 2009;50:2344-54

38 Romano I, Buchan MC, Baiocco-Romano L, et al. Physical-mental multimorbidity in children and youth: a scoping review. Dryad 2020.

39 Ontario Agency for Health Protection and Promotion (Public Health Ontario). Addressing obesity in children and youth: evidence to guide action for Ontario. Toronto, ON: Queen's Printer for Ontario, 2013.

40 Ontario Ministry of Education. Supporting minds: an educator's guide to promoting students' mental health and well-being. Toronto, ON: Queen's Printer for Ontario, 2013.

41 Pham MT, Rajić A, Greig JD, et al. A scoping review of scoping reviews: advancing the approach and enhancing the consistency. Res Synth Methods 2014;5:371-85.

42 Godin K, Stapleton J, Kirkpatrick SI, et al. Applying systematic review search methods to the grey literature: a case study examining guidelines for school-based breakfast programs in Canada. Syst Rev 2015;4:138

43 Stein RE, Bauman LJ, Westbrook LE, et al. Framework for identifying children who have chronic conditions: the case for a new definition. $J$ Pediatr 1993;122:342-7.

44 Boyle MH, Duncan L, Georgiades K, et al. Classifying child and adolescent psychiatric disorder by problem checklists and standardized interviews. Int J Methods Psychiatr Res 2017;26:e1544.

45 Kim J, Chan Y-F, McCauley E, et al. Parent-Child discrepancies in reporting of child depression in ethnic groups. J Nurse Pract 2016;12:374-80.

46 Bajeux E, Klemanski DH, Husky M, et al. Factors associated with parent-child discrepancies in reports of mental health disorders in young children. Child Psychiatry Hum Dev 2018;49:1003-10. 